\title{
Adaptive algorithm research based on micro displacement driving mirror
}

\author{
Yan Zhang \\ Changchun Institute of Optics, Fine Mechanics and Physics, Chinese Academy of Sciences, \\ Changchun 130033, China \\ 15500027661@163.com
}

Keywords: Laser communication, Facula dithering, FSM, PID, Adaptive

\begin{abstract}
Acquisition, tracking and pointing(ATP)system is the core of high speed and large capacity of laser communication in atmosphere, spot jitter generated by the disturbances among long distance laser communication has seriously affected the precise alignment between laser communication terminals, and greatly reduces the stability of the communication link and quality. In order to suppress the facula dithering, improve the accuracy of target tracking, this paper proposes an improved fast steering mirror control method. Analyzes the features of self-adaptive control and conventional PID control, and discusses the advantage of composite control with the two algorithms, proposes model reference self-adaptive control algorithm of PID, according to the Lyapunov theory, and makes algorithm simulation analysis with the conventional PID and model reference self-adaptive and PID compound control and do closed-loop tracking experiment of actual facula dithering. The experimental results show that, the adaptive PID controller obtains the overshoot is $2 \%$,rising time is $3 \mathrm{~ms}$, trackingaccuracy better than 2 microradian, overall better than the conventional PID control algorithm. Compared with the traditional PID control, control algorithm which is proposed in this paper has better inhibition of spot jitter.
\end{abstract}

\section{Introduction}

Because of the advantages of big communications capacity, high secrecy and low construction and maintenance cost, laser communication is considered to be the best solution to realize high speed and mass communication in the future ${ }^{[1]}$. To establish the communication link is the premise of laser communication, main process are capture, aiming and tracking three stages ${ }^{[2]}$. The facula dithering that caused by outside interference may cause error rate rise, seriously affect the stability of the communication link, it has become a important problems that need laser communication servo control system to solve ${ }^{[3-4]}$.

With the development of the micro displacement device, use the micro displacement with quick and high precision motion driving mirror (FSM) to compensate the disturbance is a form of suppression facula dithering. Can make the target light beam stability in the middle of the probe field, improve the position of light spot detection accuracy ${ }^{[5-7]}$. The researchers proposed feed forward compensation control ${ }^{[8]}$, control based on dynamic model ${ }^{[9]}$, inverse model compensation method based on inner product ${ }^{[10]}$ and other means to control the FSM, can achieve good control effect, but there will be some shortages, such as when the external environment changes need to be redesigned, unable to meet the requirements of the quickness of suppression facula dithering, need to know accurate mathematical model of controlled object and so on.

To make up for the lack of the control algorithm, This paper used the form of adaptive PID control based on reference model to control the FSM, the actual effect of this algorithm was verified by experiment, proved the effectiveness of the algorithm. 


\section{Principle of adaptive PID control algorithm}

\subsection{The traditional PID control system}

This article used the primary coarse tracking plus secondary fine tracking composite shaft structure to suppress facula dithering. Second control system is micro displacement driving mirror system. FSM has the characteristics of high resonance frequency, moment of inertia of small, fast response speed, small dynamic lag error and so on, is widely used in high precision laser communication. At present, the PID control is the most commonly used control method of FSM.

As the controlled object complex gradually and the control quality requirement enhances unceasingly. The disadvantages of PID control gradually emerge: generally be applied only to linear systems, can't online set controller parameters according to actual condition, need to know the control object of precise mathematical expression, difficult to implement in practical application.

\subsection{Adaptive PID control}

Aiming at the shortcomings of the traditional PID control, this paper put forward a model reference adaptive control algorithm, there is a deviation between the desired output value of the model reference and the actual output value of the controlled system in the process of control. According to the change of deviation signal and the input signal, modify the controller parameters online by adaptive laws, make the difference between the actual system output and the model output tends to zero finally. It has advantages such as setting the controller parameter automatically, simple structure, high reliability, good robustness and so on ${ }^{[1-12]}$.

\subsection{Derived Control algorithm model}

1) Algorithm's study first need to identify the mathematical model of controlled object reference, FSM is the controlled object, by experiment the resulting expression of the object as follows:

$$
G_{\mathrm{r}}(\mathrm{s})=\frac{1}{0.001 \mathrm{~s}+1}
$$

2) To calculate adaptive law is the key of the adaptive algorithm, this paper used the Lyapunov stability theory to carry on the design.

Set $r$ as the input, $k_{r}$ as adaptive controller adjustment coefficient, $y_{r}$ as the output of the reference model, $y_{a}$ as the output of the controlled object, e as the deviation. The state equations of controlled object and the reference model are:

$$
\begin{gathered}
G_{a}:\left\{\begin{array}{c}
x^{\prime}{ }_{a}=a x_{a}+c_{0} k_{a} b_{r} \\
y_{a}=c^{T} x_{a}
\end{array}\right. \\
G_{r}:\left\{\begin{array}{c}
x^{\prime}{ }_{r}=a x_{r}+c_{0} k_{r} b r \\
y_{r}=c^{T} x_{r}
\end{array}\right.
\end{gathered}
$$

$\mathrm{E}(\mathrm{s})$ :

$E(\mathrm{~s})=\mathrm{y}_{\mathrm{r}}(\mathrm{s})-y_{a}(\mathrm{~s})=\mathrm{r}(\mathrm{s})\left(\mathrm{k}_{\mathrm{r}}-\mathrm{K}_{\mathrm{s}} \mathrm{k}_{\mathrm{a}}\right) \frac{N(\mathrm{~s})}{D(\mathrm{~s})}$

In this paper, the object reference model is first order, set $\mathrm{D}(\mathrm{s})=\mathrm{Ts}+1, \mathrm{~N}(\mathrm{~s})=1$;

Equation becomes:

3) The selection of Lyapunov function

Based on the Lyapunov stability theory ${ }^{[13]}$, combined with the stability requirements of FSM, Lyapunov function as follows:

$$
\begin{aligned}
& V(\mathrm{e})=e^{2}+\lambda K^{2}(\mathrm{t})(\lambda>0) \\
& \frac{d V}{d t}=-\frac{2}{T} e^{2}+\frac{2}{T} \operatorname{Ker}(\mathrm{t})+2 \lambda K \dot{K}
\end{aligned}
$$

Set $: \quad \frac{2}{T} \operatorname{Ker}(\mathrm{t})+2 \lambda \mathrm{K} \dot{\mathrm{K}}=0$ 


$$
\left\{\begin{array}{c}
T \dot{e}+e=\left(\mathrm{K}_{\mathrm{r}}-\mathrm{K}_{\mathrm{s}} \mathrm{K}_{\mathrm{a}}\right) \mathrm{r}(\mathrm{t}) \\
K_{s}^{\prime}=\frac{e r(\mathrm{t})}{\lambda T K_{a}}
\end{array}\right.
$$

The control law:

$$
\left\{\begin{array}{c}
k_{p}=-\gamma \delta(\mathrm{t}) \mathrm{e}(\mathrm{t})-\mathrm{a}_{1}\left(\mathrm{k}_{\mathrm{p}}-\mathrm{k}^{*}{ }_{\mathrm{p}}\right) \\
k_{i}=-\gamma \delta(\mathrm{t}) \mathrm{e}(\mathrm{t})-\mathrm{a}_{2}\left(\mathrm{k}_{i}-\mathrm{k}^{{ }^{*}}{ }_{i}\right) \\
k_{d}=-\gamma \delta(\mathrm{t}) \mathrm{e}(\mathrm{t})-\mathrm{a}_{3}\left(\mathrm{k}_{d}-\mathrm{k}^{*}{ }_{d}\right)
\end{array}\right.
$$

$k_{p}^{*}, k_{i}^{*}, k_{d}^{*}$ are the initial value of PID controller, $\gamma, a_{1}, a_{2}, a_{3}$ are positive, choose the positive definite matrices as a unit array.

The tidy type:

$$
\left\{\begin{array}{l}
k_{p}=-\frac{\gamma\left(\mathrm{T}_{3}+1\right)}{1+a_{1}} e(\mathrm{t})+\frac{a_{1}}{1+a_{1}} k_{p}^{*} \\
k_{i}=-\frac{\gamma\left(\mathrm{T}_{3}+1\right)}{1+a_{2}} e(\mathrm{t})+\frac{a_{2}}{1+a_{2}} k_{i}^{*} \\
k_{d}=-\frac{\gamma\left(\mathrm{T}_{3}+1\right)}{1+a_{3}} e(\mathrm{t})+\frac{a_{3}}{1+a_{3}} k_{d}^{*}
\end{array}\right.
$$

\section{Adaptive PID control algorithm simulation}

First of all to modeling and simulation of the algorithm, divided into two parts, observe the dynamic characteristics and tracking error, according to the driving mirror disturbance signal characteristics in practical application, choose amplitude of $1 \mathrm{mrad}, 25 \mathrm{~Hz}, 50 \mathrm{~Hz}$ sine signal to simulate external disturbances as the input signal, observe the tracking error curve features. Figure 1 is a block diagram of system simulation.

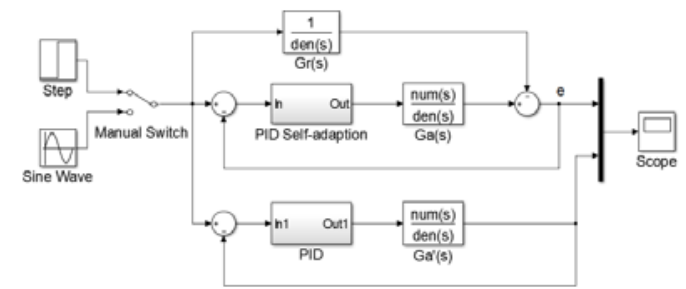

Fig.1 Simulation of system

Figure 1 contained the adaptive reference model and PID controller module. Observe the dynamic characteristics, set runtime as $0.02 \mathrm{~s}$, the simulation curve is shown in figure 2 , dotted line in figure is adaptive controller response curve, the solid line is the response curve of the PID controller.

By figure 2, when used conventional PID, the system's overshoot is about $5 \%$, rise time is about 4 $\mathrm{ms}$, when used adaptive PID, overshoot is about $2 \%$, rise time is about $3 \mathrm{~ms}$, compared with the conventional PID, this article used the model reference adaptive control can improve system dynamic performance and control effect is better.

The tracking error curve as shown in figure 3, 4, dotted lines in figure is adaptive controller tracking error, the solid line is conventional PID controller tracking error. It can be seen in the figure, the adaptive PID controller used in this paper can obtain higher tracking precision. 

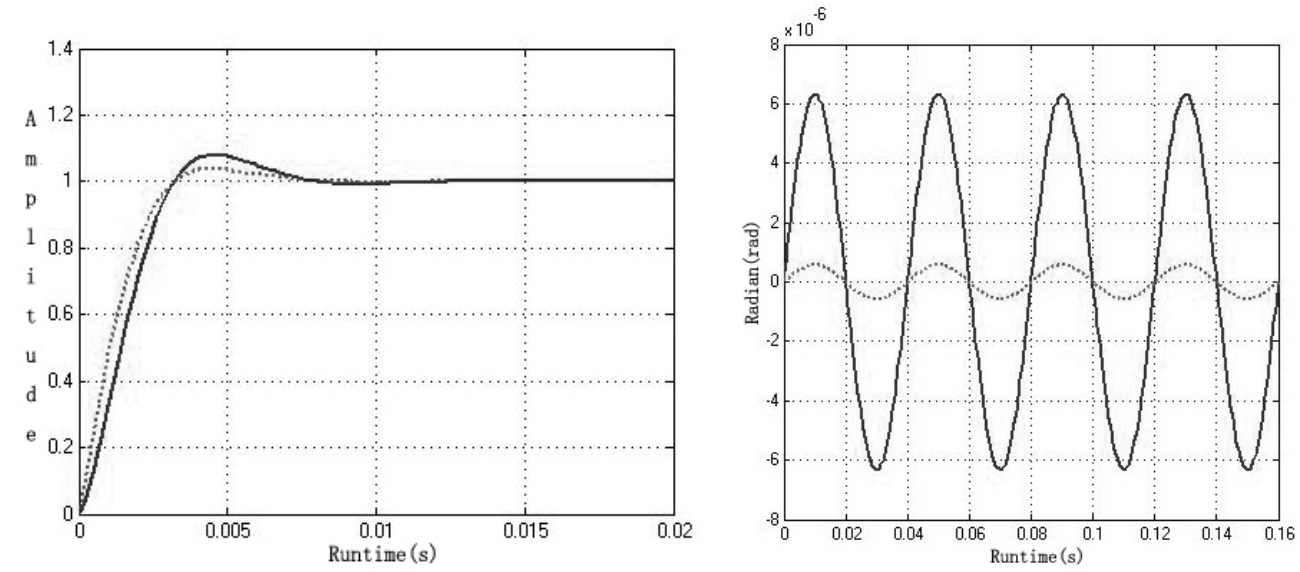

Fig.2 Phase step response of system

Fig.3 $1 \mathrm{mrad} 25 \mathrm{~Hz}$ sine signal tracking error

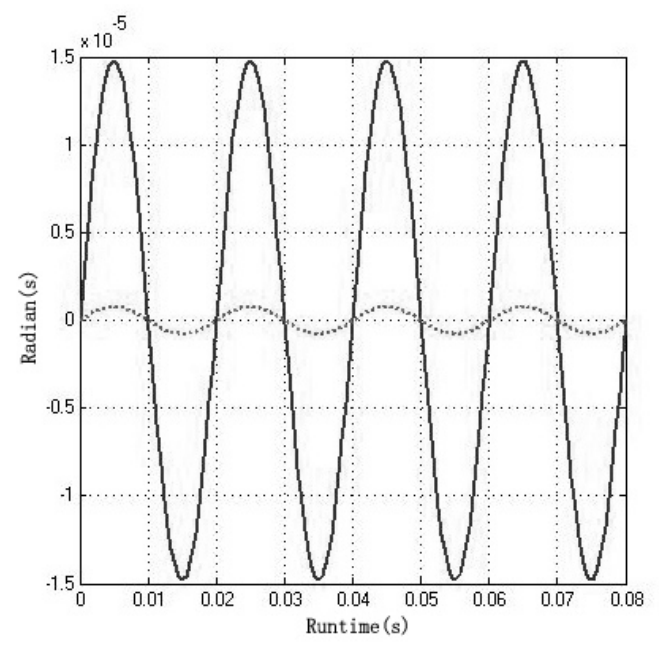

Fig.4 $1 \mathrm{mrad} 50 \mathrm{~Hz}$ sine signal tracking error

From the simulation graph we can obtained the root mean square of the tracking error. Concrete numerical value as shown in table 1:

Tab.1 Simulation tracking error

\begin{tabular}{cccc}
\hline $\begin{array}{c}\text { Amplitu } \\
\text { de(mrad) }\end{array}$ & $\begin{array}{c}\text { frequency } \\
(\mathrm{Hz})\end{array}$ & control mode & $\begin{array}{c}\text { root mean square } \\
\text { error }(\mu \mathrm{rad})\end{array}$ \\
\hline \multirow{2}{*}{1} & 25 & PID & 6.55345 \\
\cline { 3 - 4 } & \multirow{2}{*}{1} & adaptive & 0.42953 \\
\cline { 3 - 4 } & 50 & PID & 13.47937 \\
\hline
\end{tabular}

Under the same sine input signal, compared with the conventional PID controller, the tracking error that adaptive PID controller obtained has a large improvement, just as one of the points on the numerical. For adaptive controller, on the premise of the same input signal amplitude, the tracking error become larger when the signal frequency grows. When the sine input signal frequency is same, the tracking error of root mean square obtained by adaptive controller designed in this paper is better than $2 \mu \mathrm{rad}$, tracking performance is greatly superior to traditional PID controller.

\section{Curb beam jitter experimental verification}

\subsection{Build desktop experiment system}

Set up experiment system after simulation for actual test and verify, the main equipments are two pieces of FSM and its controllers, CCD camera and a computer control system. 
FSM and its porcelain drives are fabricated by PI company. The resolution is $0.02 \mu \mathrm{rad}$, closed loop trip is $2 \mathrm{mrad}$, mechanical resonance frequency with load is $1.3 \mathrm{kHz}$, repeat positioning accuracy is $0.15 \mu \mathrm{rad}$. Piezoelectric ceramic controller output voltage range is $-10 \sim 10 \mathrm{~V}$.

\subsection{Curb beam jitter experiment with adaptive PID control algorithm}

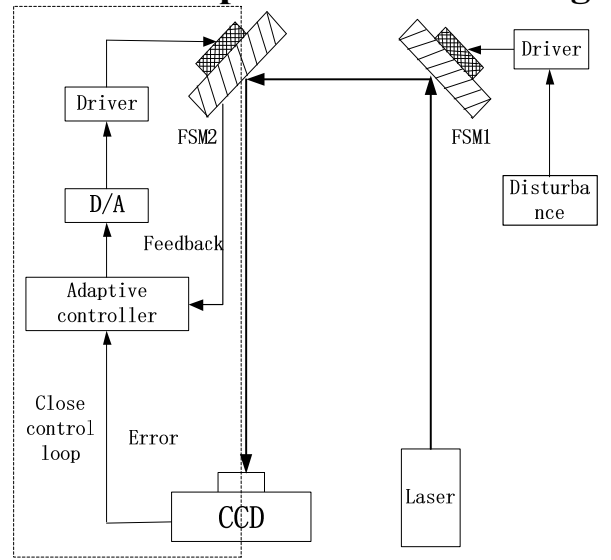

Fig.5 Experiment block diagram of system

In the experiment, control board control FSM1 to do certain frequency and amplitude of disturbance, to simulate the facula dithering caused by outside interference, the CCD camera detect the position deviation between target flare and the target surface center, transport deviation signal into controller through closed loop, adaptive controller obtains the control parameters by adaptive law according to the input signal, FSM2's drive produce rapid tiny movement according to the parameters, to reduce the amount of deviation between the flare and the target surface center, when the deviation value was zero, the controller adjust action stopped, so as to realize precise tracking of light spot. The experimental system block diagram is shown in figure 5:

FSM displacement is determined by the absolute value of the driving voltage. When set parameters and calculate the precision of tracking error, we need to convert the dimensional amount of deflection and the control voltage.

To test and verify the reliability of algorithm, choose two groups of different disturbance signal frequency, analysis the experimental data and curve fitting.

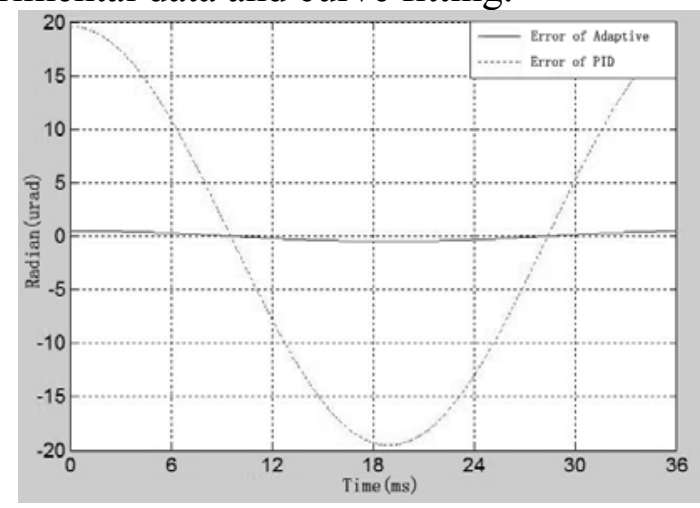

Fig.6 5V 25Hz data graph of system

When FSM1 produce sinusoidal disturbance signal amplitude is $5 \mathrm{v}$, frequency is $25 \mathrm{~Hz}$, the data curve is shown in figure 6 . 


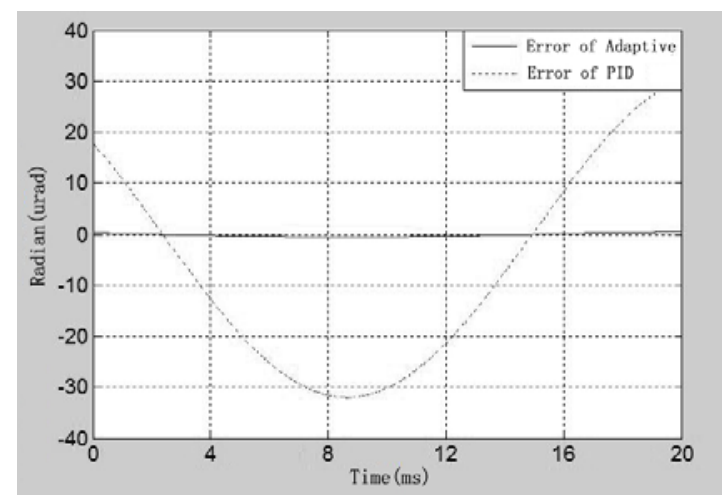

Fig.7 5V 50Hz data graph of system

When FSM1 produce sinusoidal disturbance signal amplitude is $5 \mathrm{v}$, frequency is $50 \mathrm{~Hz}$, the data curve is shown in figure 7.

It can be seen from the figure, tracking precision of the adaptive control algorithm is significantly better than conventional PID. Main leading indicators of tracking system are RMS, top-top value and isolation. Calculated parameters are shown in table 2:

Tab.2 Experiment parameters of system

\begin{tabular}{cccccc}
\hline $\begin{array}{c}\text { Amplitude } \\
(\mathrm{V})\end{array}$ & $\begin{array}{c}\text { frequency } \\
(\mathrm{Hz})\end{array}$ & $\begin{array}{c}\text { control } \\
\text { method }\end{array}$ & $\begin{array}{c}\text { error } \\
\mathrm{RMS}(\mu \mathrm{rad})\end{array}$ & $\begin{array}{c}\text { error } \\
\text { top-top }(\mu \mathrm{rad})\end{array}$ & isolation \\
\hline \multirow{2}{*}{5} & \multirow{2}{*}{25} & PID & 19.7586 & 53.3482 & 25.4576 \\
\cline { 3 - 6 } & \multirow{2}{*}{5} & adaptive & 0.68874 & 1.98796 & 54.0318 \\
\cline { 3 - 6 } & \multirow{2}{*}{5} & PID & 21.8661 & 61.6405 & 24.2027 \\
\hline
\end{tabular}

\subsection{Experimental summary}

By the experimental data, When the amplitude and frequency of the disturbance signal changed, the control algorithm being proposed in this paper can achieve good tracking performance, when the signal amplitude phase are same, the lower the frequency the better control results, when the frequency are same, the smaller the amplitude the better the results. In the numbers of experiments in this paper, the minimum RMS of the tracking error is $0.7 \mu \mathrm{rad}$, the maxima is less than $2 \mu \mathrm{rad}$, Under the condition of same disturbance signal, compared with the conventional PID control, the algorithm being proposed in this paper, RMS of the tracking error, top-top value is a small percentage of the PID controller, and isolation degree is about 50, is more than double of the conventional PID controller, having a great advantage in both tracking accuracy and stability.

\section{Conclusion}

In view of the influence of facula dithering on laser communication, This paper first discusses the causes of the facula dithering, clearly the specific problem need to be solved in this paper, propose adaptive PID control algorithm to suppress it, analysis and deduce the algorithm, determine the applicable specific algorithm, simulation model and set up the control system of the FSM desktop experiment system, verifies the algorithm combined with the actual controlled object. Compared with the conventional PID control, system rising time increased by $30 \%$, get $3 \mathrm{~ms}$, overshoot reduced nearly $60 \%$, get $2 \%$. Under the disturbance of $5 \mathrm{v}$ amplitude and $25 \mathrm{~Hz}$, precision of the tracking error is $0.68 \mathrm{rad}$, isolation is 54.0318, are much better than conventional PID control method, obviously improve the dynamic characteristic of the system and the has good inhibition effect for the facula dithering. The research and the experimental results show that, using adaptive combined with PID control method to control FSM to suppress the speckle frames, can realize high precision fast track, laid a solid foundation for realizing the laser communication system design in further research. 


\section{Reference}

[1]. Shanqiu Chen, Chao Liu, Enyi Zhao, et al. Low-cost high performance adaptive optics real-time controller in free space optical communication system[C]. Proc. SPIE. 9301, International Symposium on OptoelectronicTechnology and Application 2014: Image Processing and Pattern Recognition, 930118. (November 24, 2014)

[2]. SHAN F H, TONG SH F, LV C L. Beacon Detection Technology of APT System in Free Space Optical Communications[J]. Journal of Changchun University of Science and Technology, 2013, Z2: 53-55, 59.

[3]. LUKINVP, ORTESBV. Adaptiving and imaging in the turbulent atmosphere[M]. MALIKOVA ABtrans. SPIE, 2002.

[4]. ZHU Y L, SONG Q. Numerical simulation of the impact of atmospheric turbulence on the laser signal[J]. LASER \& INFRARED, 2013, 11: 1268-1272.

[5]. DING K, HUANG Y M, MANG Z G, et al.. Composite control fast-steering-mirror for beam jitter[J]. Opt. Precision Eng., 2011, 19(9): 1991-1998.

[6]. Myung Cho, Andrew Corredor, Christoph Dribusch, et al. Development of GMT Fast Steering Secondary Mirror Assembly [C]. Proc. SPIE. 9145, Groundbased and Airborne Telescopes V, 91451M. (July 22, 2014)

[7]. LIU B, GUO J Y,SUN Y Q. Modeling and control for PZT micro-displacement actuator[J]. Opt. Precision Eng., 2013, 21(6): 1503-1509.

[8]. Victor Skormin, Mark Tasullo. Adaptive jitter rejection technique applicable to airborne laser communication systems[C]. Proc. SPIE. 2123, Free-Space Laser Communication Technologies VI, 357. (August 16, 1994)

[9]. HEI M, LU Y F, ZHANG ZH Y, et al.. Design of fast steering mirror based on dynamics model[J]. Opt. Precision Eng., 2013, 01: 53-61.

[10]. DANG X J. Real-time adaptive inverse control based on neural networks for piezoceramic actuator[J]. Opt. Precision Eng., 2008, 16(7): 1266-1272.

[11]. WANG Q, CHENG K, FU CH Y. A method for controlling fast-steering mirror driven by voice coil motor based on the closed-loop performance [J]. Opto-Electronic Engineering, 2005, 32(2): 9-11.

[12]. Mounir Bouzoubaa, Vladimir V. Nikulin, Victor A. Skormin, et al. Model reference control of a laser beam steering system for laser communication applications[C]. Proc. SPIE.4272,Free-SpaceLaserCommunication Technologies XIII, 93. (June 20, 2001)

[13]. HAN C, BAI B X, YANG H M, et al.. Primary environment effect factors to tracking precision in space-ground laser communication[J]. Acta Photonica Sinica, 2010, 39(1): 89-94. 